\title{
Subscription Models - everything as a service
}

\author{
Extrakt des Beitrags "Subscription Models: Merkmale, \\ Praxisbeispiele und Auswirkungen auf die Unternehmensteuerung" \\ von Mischa Seiter, Lars Grünert und Kassian Kenner* erschienen in: \\ Schmalenbach IMPULSE, 1. Jg. 2021, Heft 1, \\ https://doi.org/10.54585/TSDL8165.
}

Immer mehr Unternehmen versuchen sich erfolgreich an Subscription Models. Gerade in B2C-Märkten werden regelmäßig eindrucksvolle Nutzerzahlen vermeldet. Nutzerorientierte Subscription Models bieten nicht nur Dienstleistern, sondern auch der produzierenden Industrie deutliche Vorteile. Sie stellen Anbieter aber auch vor Herausforderungen.

Unter dem Stichwort „Everything as a Service“ rollen Subscription Models immer mehr Branchen auf und sind zunehmend auch in der produzierenden Industrie zu finden. Die Bandbreite von Subscriptions Models ist in der Praxis ist groß. Allen sind vier charakteristische Merkmale gleich:

1. Es findet kein Eigentumsübergang statt. Anders als beim Kauf wird nur Zugang zu einer Leistung gewährt.

2. Es besteht eine kurze Kündigungsfrist, d. h. die Kooperation wird zunächst nur für eine begrenzte Zeit geschlossen. Der Kunde kann sämtliche Leistungsbestandteile, physische wie nicht-physische, nach dem Ende der Laufzeit zurückgeben.

3. Die Vergütung erfolgt kontinuierlich und basierend auf einer vereinbarten Zeit- oder Leistungsgröße, hier als Pay-per-X bezeichnet. Bei „X“ kann es sich um eine zeit-, nutzungs- oder ergebnisorientierte Größe handeln.

4. Es findet eine kontinuierliche Verbesserung der Leistung statt. Basis hierfür ist die Erfassung von Nutzungsdaten, um Kundenbedürfnisse zu identifizieren und passende Lösungen zu entwickeln. Darüber hinaus können Optimierungspotentiale abgeleitet werden, etwa in Bezug auf Betriebsparameter.

Prof. Dr. Mischa Seiter | Institut für Business Analytics | Universität Ulm Dr. Lars Grünert | Kaufmännischer Geschäftsführer | TRUMPF GmbH \& Co. KG

Kassian Kenner | Wissenschaftlicher Mitarbeiter | International Performance Research Institute gGmbH | KKenner@ipri-institute.com 


\section{Vorteile von Subscription Models}

Subscription Models bieten sowohl Anbietern als auch Kunden enorme Vorteile. Anbieter profitieren von tiefgreifenden Einblicken in die Kundenbedürfnisse, können ihr Leistungsportfolio optimieren und eine höhere Kundenbindung erzielen. Auf diese Weise lassen sich neue Möglichkeiten für Wertschöpfung und Wachstum erschließen. Kunden wiederum erhalten eine individualisierte, höherwertige Leistung, die ihre Bedürfnisse zielgenau erfüllt. Dabei profitieren sie zusätzlich von einem geringeren Finanzierungsbedarf sowie dem flexiblen und bedarfsgerechten Bezug der Leistung. Verglichen mit dem klassischen Produktgeschäft richten sich Subscription Models deutlich mehr nach den Kundenbedürfnissen. Nutzstiftend ist der zielführende Einsatz des Produkts, nicht dessen Eigentum.

Verglichen mit dem klassischen Produktgeschäft richten sich Subscription Models deutlich mehr nach den Kundenbedürfnissen.

In der Praxis hat bereits eine Vielzahl von Unternehmen Subscription Models etabliert. Neben Anbietern immaterieller Güter gehören dazu auch vermehrt Unternehmen der produzierenden Industrie, die im Kerngeschäft physische Güter anbieten. V.a. immaterielle Güter eignen sich gut für Subscription Models. Sie sind unbegrenzt teilbar, erzeugen vernachlässigbare Grenzkosten und unterliegen keiner physischen Abnutzung. Zudem können sie flexibel und mit geringem Aufwand in gleichbleibender Qualität zur Verfügung gestellt werden. Bekannte Beispiele sind Netflix, Apple und Walmart. Physische Güter hingegen lassen sich schwieriger als Subscription Models skalieren. Hier unterscheidet man zwei Typen: Entweder werden im Rahmen einer Subscription zusätzliche Funktionen des Kernprodukts freigeschaltet wie beim Tesla Full Self-Driving. Oder das Kernprodukt selber ist Teil der Subscription, sodass der Kunde die gewünschte Leistung als Komplettangebot erhält. Beispiele sind Light as a Service von der Deutschen Lichtmiete und TRUMPF Pay-per-Part.

\section{Herausforderungen für die Unternehmenssteuerung}

Aus den Merkmalen von Subscription Models ergeben sich spezifische Herausforderungen für die Unternehmenssteuerung. Das gilt besonders im Kontext physischer, investitionsintensiver Güter wie im Maschinenbau. Neben einem erhöhten Investitionsbedarf und veränderten Betriebsrisiken aufgrund von nutzungs- oder performancebasierter Abrechnung zählen dazu die steigende Umsatzvolatilität und ein verändertes Cash Flow-Profil. Eine zentrale Bedeutung kommt dem Lernen aus Nutzungsdaten der Kunden zu, um durch den Einsatz geeigneter Methoden, insbesondere Predictive Analytics, mit diesen Herausforderungen umzugehen.

Klassische Maschinenbauunternehmen können die erforderlichen Fähigkeiten entweder selbst aufbauen oder durch geeignete Kooperationspartner abdecken. Das Praxisbeispiel TRUMPF Pay-per-Part zeigt, wie ein Subscription Ökosystem aussehen kann, in dem verschiedene Unternehmen spezifische Fähigkeiten einbringen, die für das Angebot des Subscription Models notwendig sind. Neben TRUMPF als Maschinenbauer sind dies auch ein Finanzierungspartner (Munich Re), ein loT-Dienstleister (relayr) und ein Zulieferer (Klöckner). 
Abschließend bleibt also festzuhalten: Subscription Models sind vielfältig in ihrer Form, bieten enorme Vorteile für Anbieter und Kunden, bedürfen aber Anpassungen in der Unternehmenssteuerung. 